created. The roll-out of a national L\&D model meant that half the population of England was covered by 2015, with funding assured for a final wave of $L \& D$ services to provide for total population coverage.

Where implemented, L\&D services have been shown to lead to increased numbers of local team referrals. They may reduce court adjournments and the overall amount of time spent attending court. There is also some evidence of an association with improved mental health among both adults and young people, with reductions in re-conviction rates amongst the later. There remain deficiencies in the evidence base with regards to the economic impact of L\&D services. The majority of economic assessments of L\&D services have been performed in the United States with fewer studies in the UK.

Conclusion. Although there is evidence that liaison and diversion can produce benefits, there is a general recognition that a higher standard of evidence is required, including experimental work and assessment of economic impact. L\&D services carry a financial burden, but this may be offset by incorporating the value of the health improvements that may be brought in those who might otherwise not have received treatment.

Do reality distortions contribute to an increased risk of violent offending in schizophrenia? - a narrative review

Patrick McLaughlin

National Forensic Mental Health Services

doi: 10.1192/bjo.2021.723

Aims. To critically examine the factors that drive an increased risk of violence in the schizophrenic population, with emphasis on the role played by reality distorted symptoms.

Background. A multitude of studies have reported a positive association between schizophrenia and violence. Many of the risk factors for violence among the non-mentally disordered population, such as substance use, childhood conduct problems and victimisation, are the same as for persons with schizophrenia. There remains controversy however as to whether reality distorted symptoms themselves contribute to the increased risk of violence. Method. Relevant literature was identified through a search of the following databases: PubMed, EMBASE, and PsycINFO. Data were appraised and synthesised to provide a comprehensive overview of the current evidence base for the role of reality distorted symptoms in violence in schizophrenia.

Result. Studies ascertaining the contribution of reality distorted symptoms in violent behaviour have produced contradictory results. At a population level, several epidemiological surveys have found little or no contribution for reality distorted symptoms. Such studies frequently show that violence can be accounted for almost entirely by other factors such as substance use and victimisation. However studies investigating relationships between clinical diagnoses and population-wide violence may be unable to detect association at the symptom level. A number of studies have found strong associations between schizophrenia and violence which was not explained by comorbid substance use and have shown strong associations between specific reality distorted symptoms (in particular persecutory delusions accompanied by anger) and violent behaviour.

Conclusion. There is heterogeneity in the relationship between schizophrenia and violence. Factors that are associated with increased risk of violence among the schizophrenic population are also pertinent to those without mental disorders. With regards to the pathways to violence in schizophrenia the following conclusions may be drawn: there is an well-established increased risk of violence associated with schizophrenia which has been replicated in many studies; this risk is driven largely by substance use but other factors such as victimisation are also important; there is evidence that reality distorted symptoms, particularly persecutory symptoms, play a role in violent behaviour in some patients, particularly when co-occurring with anger; finally, there may be shared aetiological links between schizophrenia and antisocial behaviour.

\section{Mindfulness based cognitive therapy for recurrent depressive disorder}

James McLoughlin ${ }^{1 \star}$, Paula Martin², Geraldine McCarthy ${ }^{3}$ and Chee Lin Piong ${ }^{1}$

${ }^{1}$ University Hospital Galway; ${ }^{2}$ Beo Mindful Health and ${ }^{3}$ Sligo

University Hospital

${ }^{*}$ Corresponding author.

doi: 10.1192/bjo.2021.724

Aims. Mindfulness-based therapies have been demonstrated to be effective in reducing anxiety, stress and depressive symptoms in adults. Depression is a chronic relapsing condition. Major depressive disorder is one of the most common causes of ill health and functional impairment.

Our goal was to assess the real world clinical effectiveness of Mindfulness Based Cognitive Therapy (MBCT) for Recurrent Depressive Disorder in three domains:

-Depression, anxiety and stress levels

-Mindfulness level

-Self-compassion level

Method. Patients with a diagnosis of Recurrent Depressive Disorder (primary or secondary diagnosis) were referred by their community mental health team to participate in an 8-week educational MBCT programme. Participants completed the Depression, Anxiety and Stress (DASS), 5-Facet Mindfulness and Self Compassion self-rated scales prior to commencing and at the end of the course. They were also invited to give qualitative feedback at the end of the course.

Data were collected from four groups who completed the course over a period of twelve months. A paired samples test was used to compare pre and post intervention scores to determine effect size. Result. We had complete data for 19 participants out of a cohort of 34. Pre intervention scores were similar for both groups.

The mean age of the cohort was 47 years (SD of 10 years), 3 male, 16 female.

Patients showed a clinically significant reduction of symptoms in depression, anxiety and stress, with respective reductions of $48 \%$, $26 \%$ and $43 \%$ post intervention. Results were statistically significant for depressaion and stress $\mathrm{p}<0.001$ but not for anxiety $\mathrm{p}=0.130$.

Positive trends were seen in all domains of the 5-Fact Mindfulness and Self Compassions scales, with mean improvements of $28.2 \%$ and $35.3 \%$ respectively. All results were statistically significant.

We also collected anonymized qualitative feedback which highlighted themes of empowerment, skill acquisition and improved coping.

Conclusion. Numerous studies have demonstrated poor compliance with antidepressant treatments commonly prescribed in Recurrent Depressive Disorder. This small scale study demonstrates a statistical and clinical benefit of MBCT for these patients, supporting greater use of such novel non-pharmacological therapeutic options as treatment strategies.. 
To what extent does the COVID-19 pandemic impact patients with anorexia nervosa?

Krishna Mehta

Lancaster University

doi: 10.1192/bjo.2021.725

Aims. This systematic review aims to discuss the extent to which the measures undertaken to control the COVID-19 pandemic in several countries have affected those with Anorexia Nervosa (AN). The coronavirus pandemic is still raging on in many countries and its effects will still be felt years from now and previous studies have shown that it has impacted other mental illnesses. AN cases are on the rise and the nature of the illness has deadly consequences, therefore it is paramount to discuss the relationship between the COVID-19 measures and symptomatology of AN to ensure appropriate services are in place to deal with potential outcomes.

Method. Systematic search of the PubMed database gave thirtythree total results with seven of these used in this review. These studies met the inclusion criteria; examples include primary studies and use of the English Language. The exclusion criteria involved literature reviews, studies with less than ten participants and studies not separating AN from other eating disorders.

Result. Many studies were cross sectional in nature except two longitudinal studies. Anorexic symptomology increased in the majority of papers in this review. Specifically restricting intake has increased compared to before lockdown measures. Physical activity has varied on an individual level in most studies potentially due to compensatory behaviours. Co-morbid psychopathologies were also noted during these studies. There are many factors behind these changes such as food insecurity, the effect of media and social media, uncertainty and the lack of social interaction. A number of participants across the studies were dissatisfied with treatment services during lockdowns. Variability in study results may be due to the differences in measures between countries and the use of self-reported cases compared to those contacted through care services. Limitations of this review are largely due to the how current the topic is and recommendations for the future include reviewing new studies that are published and to review the situation once the full impact of COVID-19 is known. Conclusion. Patients with AN have struggled more due to the COVID-19 measures and the true effect of the pandemic is yet to be felt. Further studies and reviews should be conducted. The appropriate services should be put into place to ensure patients are supported effectively.

\section{Are drug consumption facilities an effective} intervention to reduce drug related mortality?

\section{A critical review of the literature}

Holly Melvin

University of Manchester

doi: 10.1192/bjo.2021.726

Aims. To critically appraise the literature regarding the effect of Drug Consumption Facilities in reducing overdose mortality

Drug consumption facilities (DCF) are places where people can use illicit drugs in the presence of medically trained staff, they aim to reduce fatal overdose risk, reduce risky injecting practices, and to serve as a bridge for users into mainstream treatment, healthcare and social services. Increasing numbers of fatal overdoses due to illicit drug use are a significant public health concern. The UK's statutory independent advisory body (the Advisory Council on the Misuse of Drugs) has recommended DCFs as a mechanism to reduce fatal overdoses due to illicit drugs. However, current UK legislation prohibits their provision.
Method. Systematic extraction of relevant literature from PubMed, using a search string with a focus on observational cohort studies with fatal overdose as the outcome. Appraisal of identified papers using the CASP tool

Result. 184 papers were identified, two of these met the inclusion criteria. Quality was fair/good. Neither demonstrated a clear effect of DCFs in reducing overdose mortality

Conclusion. It is difficult to draw firm conclusions due to design weaknesses and potential confounding variables. Robust design is difficult in this research area, due to lack of suitability for RCTs. Despite the lack of a clear effect on overdose mortality, DCFs may exert other positive effects and are a pragmatic and humane response to reducing risk in this target population

Prognostic evaluation of suicidality and development of risk factors in victims of physical and emotional child abuse and emotional/psychological neglect

\section{Latif Miah}

Swansea University Medical School, Morriston Hospital

doi: 10.1192/bjo.2021.727

Aims. To evaluate the effects of childhood maltreatment, specifically physical and emotional abuse and emotional/psychological neglect on the development of suicidal ideation, depressive symptoms and self-harm. It is hypothesised that there will be a strong causal link between the aforementioned types of maltreatment with suicidality, depression and self-harm.

Background. Child abuse is a major public health issue with profound developmental and mental health consequences towards victims and their contributions to society as a whole. The impact of sexual abuse is well-established, however non-sexual child maltreatment and its sequelae are not as well understood or studied.

Method. A literature search was carried out using the Pubmed, Cochrane, Scopus and Google Scholar databases. Articles were appraised according to set criteria and manually screened for relevance to the review.

Result. The results of this review demonstrate that there are statistically significant, potentially causal links between emotional and physical abuse, and emotional/psychological neglect with suicidal ideation, depressive symptoms and self-harm. More research is still required to elucidate the role of polyvictimisation in mental health outcomes and to further confirm these links between abuse and development.

Conclusion. Child maltreatment remains a large public health issue with major impact on the economy of the world. It has profound, potentially lifelong consequences on victims and is something that needs to lose its stigma so that it can be identified earlier and potential damage prevented as far as possible. The future may lie in working to remove the stigma surrounding it, standardise how it is studied and thus learn to recognise the signs earlier - ideally leading to implementation of policy to get victims to safety, preventing unncecessary harm.

How effective is clinical simulation in improving medical students' confidence when interviewing patients with mental health problems?: a comparison of pre-COVID and post-COVID medical student experiences
Philippa Mitchell ${ }^{1 \star}$, Ella Varnish ${ }^{1}$ and Arthita Das ${ }^{2}$
${ }^{1}$ Medical undergraduate, University of Sheffield, Rotherham Doncaster and South Humber NHS Foundation Trust and ${ }^{2}$ Rotherham Doncaster and South Humber NHS Foundation Trust ${ }^{\star}$ Corresponding author.

doi: 10.1192/bjo.2021.728 